\title{
ИНСТИТУЦИОНАЛЬНАЯ ЭКОНОМИКА
}

Для цитирования: Сухарев О. С. Дисфункция правил и институциональная эффективность // Журнал экономической теории. -2020 . - Т.17. — № 2. - С. 433-450

https://doi.org/10.31063/2073-6517/2020.17-2.16

УДК 330.3, 330,3

JEL: B2, L14

О. С. Сухарев

Институт экономики РАН (Москва, Российская Федерация; e-mail: o_sukharev@list.ru)

\section{АИСФУНКЦИЯ ПРАВИА И ИНСТИТУЦИОНААЬНАЯ ЭФФЕКТИВНОСТЬ}

Целью исследования является определение возможной связи институциональной эффективности, под которой понимается величина исполняемых функций на единицу трансакционных издержек, и дисфункции, понимаемой как расстройство, неисполнение некоторого числа функций, возникающее по различным причинам применительно к институтам, агентам, системам, а также отдельным функциям. Используется структурно-функциональный метод исследования, позволяющий на теоретическом уровне анализа, исходя из определения понятий, дать измерительную трактовку, а также на формальном уровне связать предполагаемое изменение изучаемых величин. Предлагается теоретическая модель связи дисфункции и институциональной эффективности, которая показывает, что возникающие расстройства функций вводимых правил могут снижать качество функционирования, но при этом будет обеспечиваться некоторая эффективность - часть правил исполняется для данного уровня издержек. Исходя из этого, частота и содержание изменения институтов влияет на качество развития, изменение уровня благосостояния как отдельных агентов, так и экономки в целом. Это влияние показывается на примере, к которому прибегал Р. Коуз при обосновании «теоремы», сформулированной Дж. Стиглером, а именно взаимодействия скотовода и фермера, когда прогон стада по посевам наносит ущерб фермеру, выступая отрицательным внешним эффектом. Тем самым рассматривается экономика двух агентов, благосостояние которых зависит от числа прогонов скота, распределения права собственности на прогон, исходной и изменяемой в дальнейшем величины институциональной эффективности и дисфункции агентов. Аналитически получен результат, что наибольшей величине институциональной эффективности не соответствует во всех случаях наименьшая величина дисфункции при рассмотренном способе определения данных параметров. Предложен метод оценки профиля дисфункции института или агента, расширяющий возможности применения функционального анализа в рамках институциональной экономической теории. Представлена возможная связь величины создаваемого взаимодействующими агентами блага и институциональной эффективности. Показано, что даже ситуация равенства нулю трансакциионых издержек не учитывает «функциональной компетенции» агентов, которая способна повлиять на эффективность используемого права собственности. Поэтому в таком случае распределение права повлияет на ценность и структуру производства. В случае ненулевых трансакционных издержек такое влияние становится определяющим в оценке динамики благосостояния и эффективности агентов контрактации.

Ключевые слова: правила, функция, дисфункция, адаптация, институциональная эффективность, коррекция институтов, благосостояние агентов, структурно-функциональный анализ

\section{Введение. Исследования по эффективности институтов $^{2}$}

В современной экономике институциональные факторы и среда, образуемая институтами,

\footnotetext{
1 с С Сухарев О. С. Текст. 2020.

Термины «институциональная эффективность» и «эффективность институтов» в статье употребляются равнозначно.

${ }^{2}$ Конечно, во введении одной статьи невозможно отразить полный набор и различные подходы к исследованию ин-
}

сильно влияют не только на интенсивность экономических изменений, но и на качество жизни и темп экономического роста. Наравне с мерами бюджетной и денежно-кредитной политики правительства разных стран всё чаще приходят к необходимости реформирования экономической системы, замены правил либо

ституциональной эффективности, однако наиболее общих два вектора, которых придерживаются различные исследователи, будут выделены. 
существенной их модификации, то есть к проведению институциональных коррекций как метода экономической политики. При этом последствия многочисленных институциональных изменений предсказать довольно проблематично, так как изменение правила не гарантирует целевого результата и само по себе не может быть целью. Обычно правила изменяют для того, чтобы достичь некой содержательной цели развития, исходя из того предположения, что новые правила позволят это сделать. Кроме того, пока происходит изменение, до тех пор, пока оно не завершено, вообще трудно сказать, к чему оно может привести, тем более, когда изменяются одновременно не просто отдельные правила, а целые наборы правил, регулирующих различные аспекты человеческой жизни. Эффективность самой замены зависит не только от исходной эффективности действовавшего правила, а также его окружения, но и от взаимодействия с другими изменяемыми и продолжающими действовать правилами.

Исследовать состояние объекта можно по тому, как на него действуют правила - новые и старые, а также нужно учитывать изменение состояния объекта при смене правил, оценивая их эффективность, что обычно делается в институциональной экономике по величине трансакционных издержек, генерируемых агентами, следующими в той или иной степени данным правилам. В этом случае правила рассматриваются как данность, статично. Разумеется, издержки возникают при замене или модификации правил, так что сама замена правил может не понизить в ближайшее время, а наоборот, увеличить трансакционные издержки, но если далее они будут понижаться, то такой проект институциональной трансформации может стать выгодным и обоснованным. Проблематика смены правил исходя из конкретных задач развития экономики или изменения состояния объекта может составить отдельное направление в институциональных исследованиях. В этом случае интерес представляет состав правил, их взаимодействия, очерчиваемые функции и т. д. Эффективность институтов динамически изменяется, поэтому в статике их влияние уже описать довольно трудно и нужно учитывать динамическое изменение этой эффективности во влиянии на рассматриваемые объекты и подсистемы экономики, которые также изменяются. Это более сложное направление исследований, причем важно учитывать не только количественную сторону изменения эффективности институтов, например, по трансакционным издерж- кам, но и влияние на другие виды издержек - трансформационные, а также то, что происходит с качественным содержанием функционирующего института. Функциональный набор правил должен рассматриваться в рамках функционального анализа, в аппарат которого требуется включить изучение дисфункций как особых состояний правил, при данных значениях их эффективности. Связь стандартной эффективности правил, даваемой по изменяемой величине трансакционных издержек и дисфункции правил, при этом составляет важную задачу изучения природы и содержания институциональных изменений, с возможными полезными выводами для политики проведения институциональных коррекций.

Экономические институциональные исследования можно разделить на две большие группы.

К первой относятся те, которые устанавливают влияние институтов на эффективность функционирования организации, фирмы и т. д. Они исходят из того, что действующие правила относительно экономических событий и организаций стабильны и детерминируют текущую их эффективность. Например, исследуется влияние институтов на антимонопольную эффективность, а конкретно независимость работы и эффективность антимонопольной службы (Мa, 2010). Для телекоммуникационной отрасли определяется влияние институциональной среды на ее развитие и реформирование, в частности, юридическая полнота за счет приватизации (Mohamad, 2014). Некоторые исследователи определяют аспект «структурной эффективности» (Yang, Jung, 2016) или институциональной структуры на эффективность работы банковского сектора (Chan, Koh, Zainir, Yong, 2015), в частности, влияние концентрации на эффективность банков. В рамках этой группы исследований изучается влияние политических институтов на работу правительства и его эффективность (Bartolini, Santolini, 2017), качества институтов торговли в виде ограничений на динамику производительности (Beverelli., Fiorini., Hoekman, 2017). Проблема «ресурсного проклятия» в макроэкономическом аспекте подается так, что при избыточности ресурсов эффективность их использования ниже, чем при недостатке. Это требует ввода других институтов, способных изменить подобное соотношение и повысить эффективность использования ресурсов (Hartwell, 2016). В данном случае правила трактуются также как стабильные, речь ведется о вводе новых правил, но делается оговорка, что 
нужно рассматривать этот ввод как совершенствование действующих институтов. Тем самым явно или неявно, но возникает проблема эффективности самих правил, подаваемая в аспекте их совершенствования (как потребности). Первая группа исследований многочисленная, включает огромное число прикладных работ, затрагивающих ту или иную отраслевую специфику. Кстати, обычно влияние правил, регулирующих различные секторы, друг на друга, а важность такого воздействия исключить нельзя, в подобных исследованиях не принимается во внимание. Изучение работы институтов отдельных видов деятельности, отраслей выполняют для подтверждения тех или иных теоретических позиций, иногда гипотез, но сами эти позиции, как правило, не формулируются. Вместе с тем оценка качества институтов, среды, регулирования обычно базируется на небезупречных методиках, причем с использованием разных процедур можно получить отличающиеся результаты. В экономике стабильность правила является весьма сильным допущением, поскольку правила могут быть стабильны, но расстроены, либо низкоэффективны, что скажется на функционирующих агентах и системах. Эффективность функционирующих объектов (в силу действия тех или иных правил) и институциональная эффективность, а также состояние правил (качественные характеристики), обнаруживают эффект взаимной детерминации.

Поэтому ко второй группе исследований, которая явно меньше по числу работ относительно первой группы, можно отнести именно те, где рассматривается эффективность правил, например, делается вывод о низкой величине эффективности и предлагается замена институтов по этой причине. Такие исследования предполагают в качестве предмета рассмотрения действующие правила, оценку их эффективности, на мой взгляд, обладают большей теоретической, нежели прикладной значимостью. Например, в одной из таких работ исследуется «lock-in» состояние институтов и их эффективность (Khalil, 2013), то, как возникают и изменяются правила (институты) вследствие кризисов и адаптации агентов к таким изменениям. Рассмотрение вопросов институциональных изменений можно также отнести к данной группе работ, поскольку изучаются причины изменений, собственно трансформация институтов, взаимодействие формальных и неформальных правил (Leeuw, Gössling, 2016). Анализ обращен к сравнению альтернативных траекторий и модельных ва- риантов, собственно затрагивающих «содержание институтов». В них, как правило, вопрос эффективности институтов в динамике с учетом изменения состояния не рассматривается, но оценке подлежат различные виды институциональных изменений и дизайна, реакции агентов - оппорнутистов на ту или другую модель институциональных изменений (Mahoney, Thelen, 2010; Davanzati, 2018). Важны механизмы изменений, причем с учетом технологий в вебленовском понимании (Веблен, 2007, 2010), а также с поиском ответа на вопрос о причинах изменения или сохранения институтов, особенно при изменении информации и знаний (Eggertsson, 2009). Эти темы не могут не затрагивать аспекта эффективности и дисфункции институтов, поскольку не только при трансплантации правил из одной социальной среды в другую или организационных изменениях (Полтерович, 2001; Клейнер, 2016; Сухарев, 1999) возникает явление дисфункции. Оно охватывает работающие институты с различной степенью даже при отсутствии указанных изменений, то есть институты, подобно человеческому организму, со временем накапливают некоторый объем отклонений - дисфункций, выражающихся в потере полезных свойств и функций, которые были приданы этим правилам изначально - при возникновении или вводе в социальную среду. Причиной увеличения дисфункции правил может быть конфликт правил (в силу недостатка или избытка правила), эффект переноса правила, ввода нового правила, изменения агентских представлений о правиле и т. д. и т. п. Поскольку в институциональном анализе накопилось довольно много дискуссионных моментов относительно представлений об эффективности и устойчивой неэффективности норм (ловушка), эффекте блокировки («lock in») и дисфункции, актуальной задачей является модельный ввод и демонстрация связи эффективности института ${ }^{1}$ и его дисфункции. С одной стороны, институциональную эффективность принято давать по экономии трансакционных издержек (Coase, 1994, Williamson, 2002), связанных с функционированием правил, но, с другой стороны, расстроенные правила, неисполняемые правила могут не приводить к значительной величине трансакционных издержек, следовательно, по формальному критерию эффективности быть

\footnotetext{
${ }^{1}$ Не только института, под которым понимается формальное правило, но и, скажем, экономической системы, в частности, экономики, представленной двумя агентами - фермером и скотоводом, как в задаче Рональда Коуза, проясняющей действие его теоремы (Coase, 1988, 1994).
} 
относительно эффективными, но при этом подверженными дисфункции. Если вести речь о неэффективной устойчивой норме, то, видимо, требуется пояснить, в чем эта устойчивость состоит, измеряется ли они временем неэффективного состояния или каким-либо иным показателем. Кроме того, как за некоторый период будет изменяться сама неэффективность (Полтерович, 2001), влияет ли дисфункция на эффективность/неэффективность и какова возможная связь функционального разнообразия и эффективности. При наличии запирающего эффекта норма все равно продолжает исполнять некоторые функции, причем не все из них могут исполняться с «отрицательным качеством».

Иногда рассмотрение институциональной эффективности происходит неявно, когда речь ведут о замене институтов, не акцентируя проблему эффективности действующих институтов, в других случаях само исследование может быть посвящено эффективности действующих правил с демонстрацией того, что эти правила требуют замены. При этом вопрос эффективности правила, оценки, измерения может отойти на второй план и исследователь сосредотачивается на том, что эффективность объекта могла быть выше, если бы правила были иными. Следует ли из этого, что именно институциональная неэффективность является причиной такого функционирования объекта, либо присутствуют иные причины? Может быть, причиной неудовлетворительного функционирования является дисфункция по основным функциям объекта, системы, а отнюдь не низкая эффективность работы правил. Причиной может быть и дисфункция институтов. Тогда возникает вопрос, стоит ли считать низкоэффективное функционирование дисфункцией (наибольшая дисфункция), а относительно высокую эффективность связывать с отсутствием дисфункции (наименьшая дисфункция)? Иными словами, можно ли отождествлять неэффективность и дисфункцию? Представим ниже теоретический модельный анализ, который показывает, что подобное отождествление не будет обоснованным с позиции содержания одного и другого понятия. Наибольшая дисфункция может не отвечать минимальной эффективности институтов, как и наибольшая эффективность - соответствовать минимальной дисфункции правил.

\section{1. Эффективность и дисфункция правил}

Экономика в своем функционировании подчиняется набору установленных правил, как и сам процесс воздействий, призванных обеспечить движение системы в направлении поставленной цели, которая в свою очередь также выступает своеобразным правилом. Эти формальные правила - институты (нормы, законы, приказы, постановления) задают возможности развития экономических систем (North, 1990, 2005, 2008; Hodgson, 2010).

Эффективность функционирования экономической системы обычно представляется как достижение целей ее развития, результата на единицу осуществленных затрат, с помощью которых и достигаются данная цель и результат. При этом введенные правила, которым агенты системы могут следовать либо нарушать их, определяют возможности как по затратам, так и по достижению планируемого результата. Экономические системы, как и правила, на которых они построены, исполняют определенные функции, причем правила вводятся для того, чтобы обеспечить выполнение этих функций. Однако на практике функции могут не выполняться, число полезных функций, в которых существует потребность, может изменяться. Подобное состояние, когда наблюдается расстройство функций по разным причинам, можно рассматривать как дисфункцию правил (Сухарев, 1999, 2001). Она может происходить в силу избытка функций или необоснованного замещения одних правил другими, которые при их вводе кажутся более целесообразными и эффективными, либо по причине имманентных качеств действующих правил, приспособительной реакции агентов на них, стремящихся обойти установленные правила (в силу возросших трансакционных затрат) и т. д.

В социологическом функциональном анализе термин «дисфункция» использовался Р. Мертоном (Мертон, 2006. С. 146) в значении последствий, «которые уменьшают адаптацию или регулировку системы». В противоположность дисфункции, наоборот, функции способствуют адаптации, приспособлению. Примерно такое же видение закрепилось об адаптивной эффективности институтов у Д. Норта (North, 1990). Вместе с тем агенты способны адаптироваться - приспособиться к ситуации, когда функции утеряны, расстроены, избыточны либо быстро изменяются, что и сопровождается их дисфункцией. Даже при дисфункции какого-то института его функциональный потенциал не весь может быть расстроен, и какая-то часть функций будет исполняться с необходимым или приемлемым качеством и при приемлемых трансакционных издержках. 

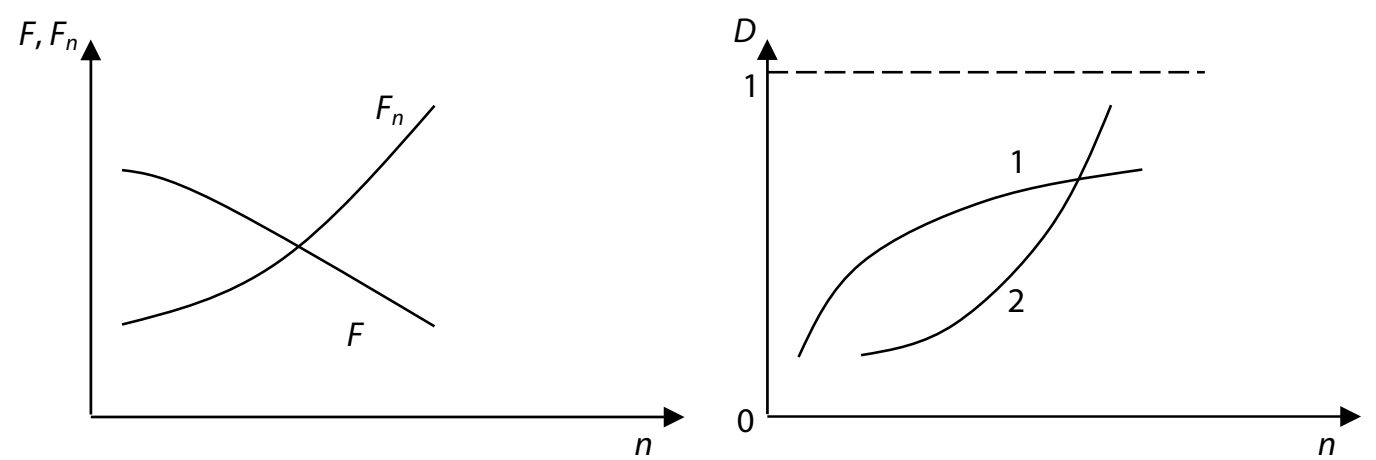

Рис. 1. Функции $\left(F, F_{n}\right)$ и дисфункция (D) от скорости институциональных изменений (n)

Поэтому важно то, какое число функций исполняется, какое не исполняется из имеющегося набора. Дисфункция может быть определена структурой исполняемых и неисполняемых (расстроенных, потерянных) функций. Для количественной стороны определения дисфункции причины возникновения или углубления уже отходят на второй план, хотя для предотвращения дисфункций, снижения числа и глубины они имеют значение.

Степень дисфункции правил может быть различная, является динамическим параметром. Эффективность правил - институциональная эффективность может быть определена как отношение числа исполняемых функций на единицу трансакционных затрат (Tr). При реализации большего функционального набора $^{1}$ с меньшими затратами эффективность такого института выше. При управлении имеются полностью исполняемые функции и частично исполняемые или неисполняемые. Тогда общее число функций $\left(F_{\mathrm{o}}\right)$ данного набора правил можно обозначить как сумму исполняемых $(F)$ и неисполняемых функций $\left(F_{n}\right)$, то есть $F_{\mathrm{O}}=F+F_{n}$.

Институциональная эффективность по исполняемому числу функций составит $E_{I}=F / T r$. Общая институциональная эффективность будет $E_{I}^{\mathrm{O}}=F_{\mathrm{O}} / \operatorname{Tr}$. Несложно показать, что общая институциональная эффективность складывается из институциональной эффективности по исполняемым и неисполняемым функциям $\left(E_{I}^{n}\right)$, то есть $E_{I}^{\mathrm{O}}=E_{I}+E_{I}^{n}$, где $E_{I}^{n}=F_{n} / T r$. Дисфункция правил $(D)$ может быть определена в таком случае как отношение числа неисполняемых функций (расстроенных функций) к общему числу функций, то есть $D=F_{n} / F_{0}$. Понятно, наибольшая дисфункция равна единице, когда расстроенные функции равны общему числу функций данного правила или си-

\footnotetext{
${ }^{1}$ Под функцией понимаем исполнение, совершение, осуществление чего-либо, обязанность что-то выполнить, под дисфункцией -расстройство, неисполнение функции либо исполнение со сниженным качеством.
}

стемы правил (институциональной системы). Наименьшее значение дисфункции равно нулю, когда расстроенных функций нет, $F_{n}=0$, $D=0$. Таким образом, для данной процедуры измерения дисфункция изменяется от нуля от единицы.

При росте числа неисполняемых функций дисфункция правила увеличивается. Если растет скорость институциональных изменений, агентам недостаточно времени для адаптации к изменяемым правилам, в связи с чем нарастает уклонение от их исполнения, увеличивается дисфункция (см. рис. 1).

В зависимости от частоты смены институтов, ввода новых правил изменяются приспособительные реакции агентов, при росте такой скорости число исполняемых функций может снижаться, неисполняемых - возрастать (см. рис. 1 , слева). Данная ситуация отвечает росту дисфункции правил, но само изменение дисфункции может происходить по-разному, согласно кривой 1 или 2 на рисунке 1 , справа. Быстрота роста может отличаться и задаваться качеством самих правил (институтов), моделями адаптации агентов и прочими институциональными параметрами, включая масштаб отторжения правил действующими институтами, исходную величину имманентной дисфункции и величину скорости изменения правил, а также содержания правил.

Чем выше уровень неисполнения правил, расстройства институтов, тем ниже адаптация, особенно если этот уровень растет (рис. 2 , слева). Устойчивость правил в большей степени способствует адаптации к ним, нежели увеличивающееся расстройство правил. Чем выше интенсивность изменения правил (институтов), тем также ниже уровень адаптации (рис. 2, справа)

Конечно, адаптация к институциональным изменениям зависит от многих иных факторов, включая качественный состав агентов, и в некоторых случаях с ростом числа изменений она может и возрасти. Однако, как правило, 

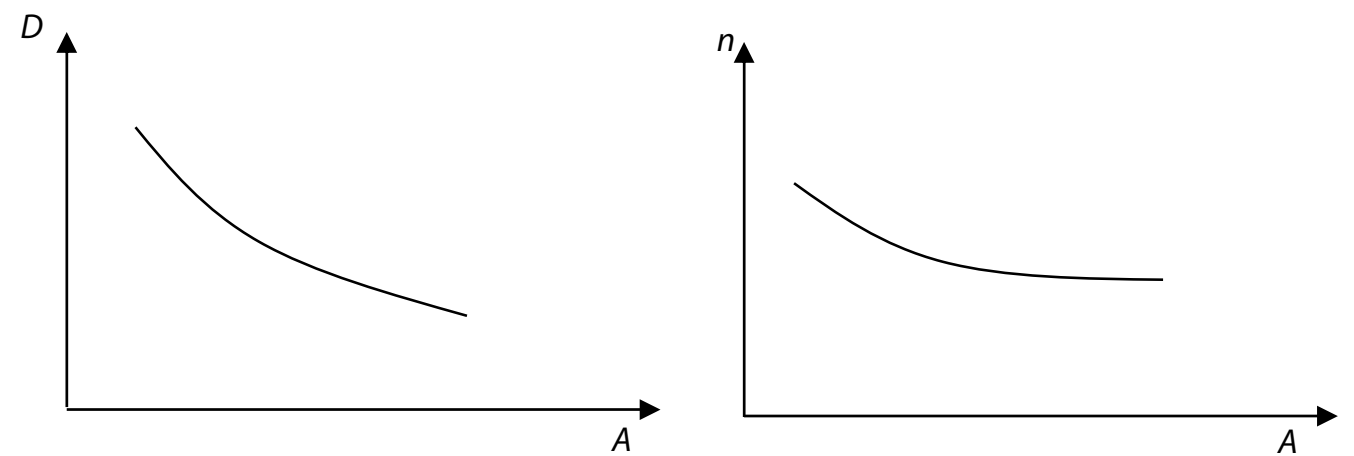

Рис. 2. Адаптация (A), дисфункция (D) правил и частота их изменения (n)

быстрая и частая смена правил дезориентирует агентов, повышает издержки и ухудшает адаптацию.

Дисфункция правил связана с эффективностью их исполнения. Исходя из приведенных выше соотношений, запишем: $E_{I}=F / T r=$ $=\left(F_{0}-F_{n}\right) / T r$, учтя, что $D=F_{n} / F_{0}$, получим: $E_{I}=$ $=(1-D) F_{0} / T r$. Институциональная эффективность снижается при росте трансакционных издержек и снижении числа исполняемых функций. При максимальной дисфункции $D=1$ институциональная эффективность равна нулю. Рост дисфункции при росте числа неисполняемых функций будет снижать институциональную эффективность. Трансакционные издержки также, видимо, будут расти с ростом числа неисполняемых функций $\operatorname{Tr}=g\left(F_{n}\right)$, что при стабильной величине общего числа функций $F_{0}=$ const рост трансакционных издержек также будет действовать в направлении снижения институциональной эффективности. Отсюда запишем условия снижения (1) и повышения (2) институциональной эффективности:
1) $\frac{d E_{I}}{d t}<0, \frac{d T r}{d t}>0, \frac{d F}{d t}<0$;
2) $\frac{d E_{I}}{d t}>0, \frac{d T r}{d t}<0, \frac{d F}{d t}>0$.

Условие (2) - это условие повышения институциональной эффективности, которое с учетом $E_{I}=F / T r$ при дифференцировании можно представить в следующем виде: чтобы институциональная эффективность возрастала, необходимо, чтобы:

1) $E_{I}<d F / d T r$, при $d T r / d t>0$, то есть при росте трансакционных издержек;

2) $E I>d F / d T r$, при $d T r / d t<0$, при снижении трансакционных издержек.

Введя коэффициент исполнения функций $k_{I}=F / F_{0}$ и рассматривая рост институциональной эффективности как основополагающее исходное условие развития правил $d E_{I} / d t>0$, из записи $E_{I}=(1-D) F_{0} / T r$, дифференцируя институциональную эффективность по времени, окончательно получим: чтобы возрастала институциональная эффективность $\left(d E_{I} / d t>0\right)$, изменение дисфункции должно быть меньше разницы темпов изменения общего числа функций и трансакционных издержек, взвешенной на коэффициент исполнения функций:

$$
\frac{d D}{d t}<\left(g_{F_{\mathrm{O}}}-g_{T r}\right) k_{I}, g_{F_{\mathrm{O}}}=\frac{1}{F_{\mathrm{O}}} \frac{d F_{\mathrm{O}}}{d t}, g_{T r}=\frac{1}{T r} \frac{d T r}{d t} .
$$

При снижении трансакционных издержек институциональная эффективность по исполняемым правилам должна быть выше, чем $d F / d T r$. Полагая, что $F=F_{\mathrm{O}}-D \cdot F_{\mathrm{O}}=(1-D) F_{\mathrm{o}}$, можем переписать условие на институциональную эффективность при ее увеличении:

$$
E_{I}>(1-D) \frac{d F_{\mathrm{O}}}{d T r}-\frac{d D}{d T r} F_{\mathrm{O}} .
$$

Поскольку общая институциональная эффективность определяется выражением $E_{I}^{\mathrm{O}}=E_{I}+E_{I}^{n}$, то есть равна сумме эффективности по исполняемым и расстроенным функциям, выражение для дисфункции, чтобы общая эффективность росла $d E_{I}^{\mathrm{O}} / d t>0$, принимает вид:

$$
\frac{d D}{d t}<\left(g_{F o}-g_{T r}\right) k_{I}+\frac{\operatorname{Tr}}{F_{\mathrm{O}}} \frac{d E_{I}^{n}}{d t} .
$$

Допустим, что изменение институциональной эффективности $d E_{I} / d t=z$, некой положительной константе, тогда изменение дисфункции будет равно:

$$
\frac{d D}{d t}=\left(g_{F_{\mathrm{O}}}-g_{T r}\right) k_{I}-z \frac{T r}{F_{\mathrm{O}}}, k_{I}=1-D .
$$

Теперь исследуем институциональную эффективность по исполняемым функциям и дисфункцию на экстремум, то есть, $d E_{I} / d t=0$, $d D / d t=0$. Уже понятно, что наибольшей дисфункции, равной единице, отвечает самая низкая институциональная эффективность $\left(E_{I}=0\right.$, нет исполняемых без расстройства функций), но какая дисфункция отвечает наибольшей эффективности, предстоит получить. 
Исходя из $d E_{I} / d t=0$ имеем $d D / d t=(1-D) \times$ $\times\left(g_{F_{\mathrm{O}}}-g_{T r}\right)$. Интегрируя с постоянной $C$, получаем дисфункцию в точке экстремума $E_{l}$, равную: $D=1-\left(\operatorname{Tr} / F_{\mathrm{O}}\right) e^{C}$. Откуда, подставляя в выражение $E_{I}=(1-D) F_{\mathrm{o}} / T r$, получаем, что $E_{I}=e^{\mathrm{C}}$. Дисфункция при этом явно не максимальная, то есть меньше единицы. При $D=1, d D / d t=0$, однако, исследуем выражение для дисфункции $D=1-F / F_{\mathrm{o}}$ на экстремум. Получим $d F_{\mathrm{o}} / F_{\mathrm{o}}=d F / F$, интегрируя с постоянной $B$, получаем, что $F_{\mathrm{O}}=F e^{B}$. Откуда дисфункция в точке экстремума $D=1-1 / e^{B}$. Это также ниже максимального значения. Для рассматриваемой задачи это значение дисфункции, для которой институциональная эффективность $E_{I}=\left(F_{\mathrm{O}} / T r\right) \times$ $\times\left(1 / e^{B}\right)$.

Таким образом, получаем:

I) $d E_{I} / d t=0$, при $D=1, E_{I}=0$; при $D=1$ $-\left(\operatorname{Tr} / F_{\mathrm{O}}\right) e^{\mathrm{C}}, E_{I}=e^{\mathrm{C}}$.

II) $d D / d t=0$, при $D=0, E_{I}=F_{0} / T r$, при $D=1, E_{I}=$ $=0$, при $D=1-1 / e^{B}, E_{I}=\left(F_{\mathrm{O}} / \operatorname{Tr}\right)\left(1 / e^{B}\right)$.

Также несложно заметить, что $E_{I} / E_{\mathrm{O}}=1-D$, что отражает рисунок 3 , слева. В правой части рисунка показан возможный вариант связи дисфункции и институциональной эффективности.

Левая часть рисунка 3 отражает долю институциональной эффективности в общей величине эффективности $E_{0}$, которая понижается по мере роста дисфункции института. В правой части рисунка 3 показан теоретический вариант связи дисфункции и институциональной эффективности $E_{I}$, причем характер связи определяется движением (эволюцией) системы по стрелочкам, но наибольшей эффективности не соответствует наименьшая дисфункция. Ниже представленный расчет, согласно введенным соотношениям, подтверждает также именно такое соотношение. Безусловно, при проведении институциональных исследований в конкретных полевых условиях возможно возникновение своего результата. Однако то, что наибольшая эффективность, видимо, соответствует не мак- симальной, а меньшей степени дисфункции, но и не самому низкому значению, остается справедливым для целого набора практических примеров. Институциональная эволюция по стрелке $k_{1}$ связана с тем, что с ростом эффективности института дисфункция снижается (интенсивный рост институциональной эффективности). Затем снижение эффективности может сопровождаться снижением дисфункции, например, при замене или модификации данного правила - будет низкой и его эффективность, и дисфункция. При движении по стрелке $k_{2}$ с ростом эффективности может возрасти и дисфункция (экстенсивный рост институциональной эффективности). В дальнейшем снижение эффективности может сопровождаться ростом дисфункции.

Рассмотрев полученные соотношения и приняв в качестве одного из вариантов правдоподобное условие, что $\left(\operatorname{Tr} / F_{\mathrm{O}}\right) e^{C} e^{B}>1$, отразим на рисунке 4 возможную связь величины дисфункции и институциональной эффективности, исходя из расчетного примера, исследуя выражения для институциональной эффективности и для дисфункции на экстремум $d D / d t=0, d E_{I} / d t=0$. Конечно, кривые нарисованы произвольно, но характерные точки отвечают полученным соотношениям при исследовании указанных функций на экстремум (приведены выше - позиции I-II по тексту).

Проведенный анализ позволяет утверждать, что линейной связи между дисфункцией и институциональной эффективностью не обнаруживается. Наибольшая дисфункция, когда, так или иначе, расстроены (не соблюдаются) все правила, отвечает нулевой институциональной эффективности. Однако при нулевой дисфункции институциональная эффективность имеет некоторое значение, которое выше всех прочих значений для любых дисфункций в диапазоне от нуля до единицы - JS. Вместе с тем имеется участок, где эффективность институтов может быть еще выше для небольшого диапазона значений дисфункции - SH, где дисфункция мала
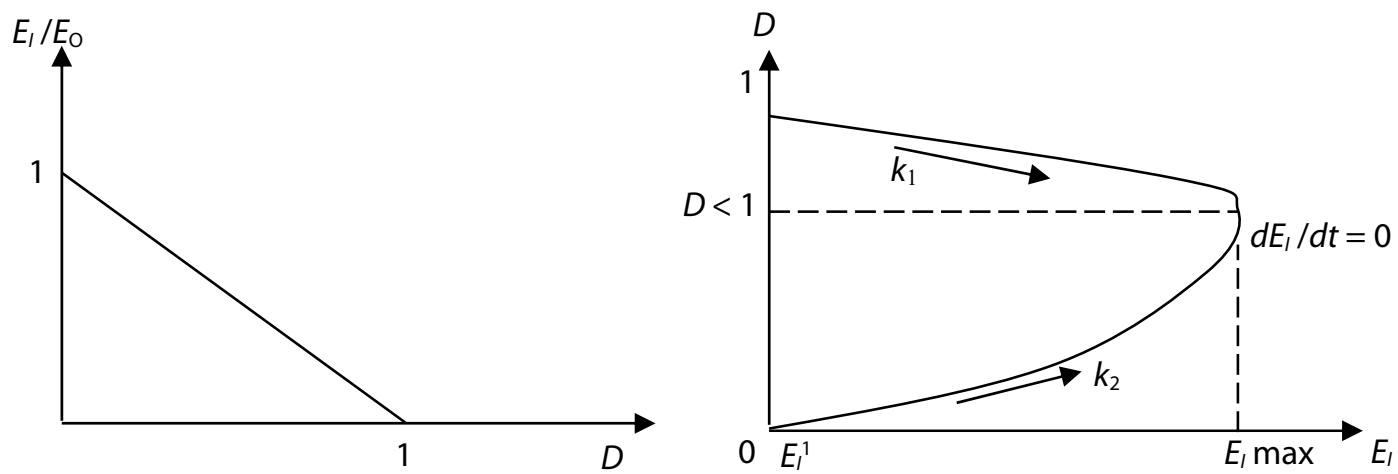

Рис. 3. Дисфункция и институциональная эфффективность - различные варианты 

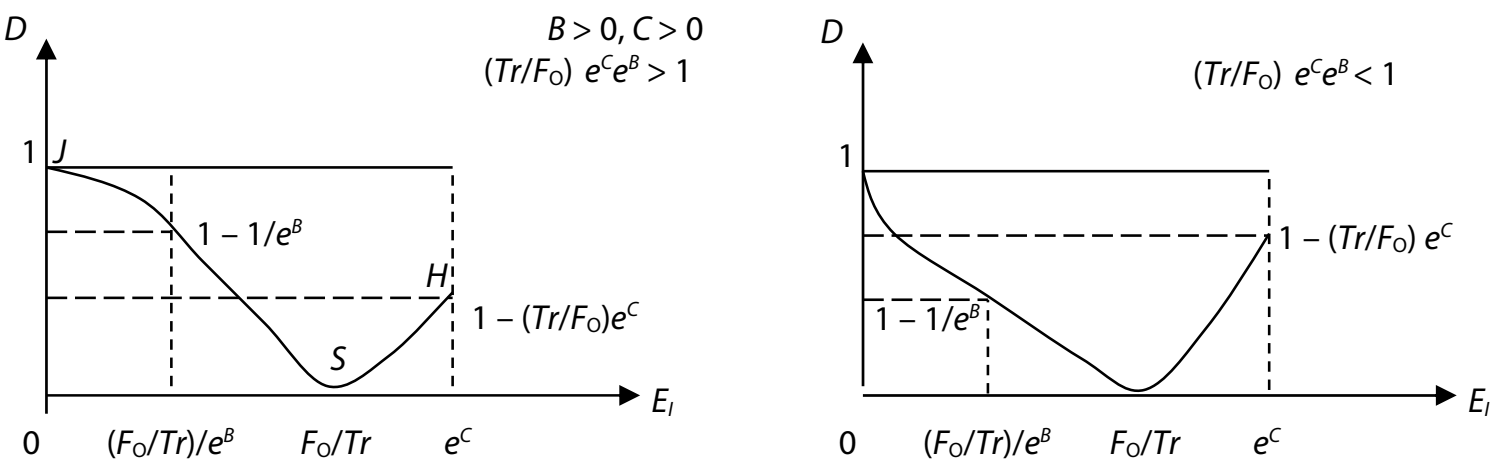

Рис. 4. Дисфункция и институциональная эффективность

(рис. 4, слева). Снижение дисфункции на этом участке может снизить и институциональную эффективность, а повышение институциональной эффективности - увеличить дисфункцию, но ее значение на этом участке невелико. Подобные связи на практике довольно сложны и затрудняют управление системой. При принятии решения редко учитывают то, насколько могут быть расстроены функции, какое их число необходимо, почему агенты перестанут исполнять часть функций, и насколько сами функции полезны развитию. На рисунке 4 справа показана ситуация при $\left(\operatorname{Tr} / F_{\mathrm{O}}\right) e^{C} e^{B}<1$. Большей институциональной эффективности соответствует и большая дисфункция, если сравнивать с ситуацией на рисунке 4 слева. При других значениях констант $B, C$, скажем, меньше нуля, изменится соотношение значений по оси абсцисс $E_{r}$. Вид кривой будет похожий, хотя участок $S H$ будет отличаться, большей институциональной эффективности будет соответствовать либо меньшая, либо большая дисфункция. Результат на рисунке 4 слева более приемлем, нежели справа, так как при той же величине институциональной эффективности величина соответствующей ей дисфункции ниже, чем на рисунке 4 справа.

Представленный анализ особо важен при проведении институциональных коррекций системы, например такой, как научная сфера, функционирующая в перманентном режиме смены либо модификации правил, что не может не сказываться на адаптации и эффективности ее функционирования. Одна из задач поиска мер воздействия на различных агентов экономики состоит в том, чтобы определить, насколько изменяемые правила будут эффективны и какое число полезных функций будет выполняться, если следовать этим правилам, почему одни агенты будут следовать правилам, но другие им следовать не будут, что и порождает дисфункцию. Рассмотрим экономическую систему, состоящую из двух агентов, оценив возможное изменение ее благосостояния при различном функциональном состоянии.

\section{2. Экономика двух агентов: функциональный анализ}

Сочетание институтов, регулирующих развитие в каждом случае, будет давать отличающуюся степень дисфункции институтов, а разные виды воздействий агентов друг на друга и команды принуждения в рамках системы управления - задавать различный уровень агентских взаимодействий, их эффективности и дисфункции. Если для данного института какой-то набор функций из имеющегося числа не исполняется на должном уровне либо в принципе, и это становится неотъемлемой характеристикой функционирования данного института $\left(\right.$ нормы $\left.^{1}\right)$, то можно говорить о его устойчивой дисфункции. Величина самой дисфункции может оказаться небольшой, но может быть и значительной. Такую норму можно считать устойчивой дисфункциональной, с разной степенью дисфункции. Однако эффективность при этом может изменяться. Норма может не оказаться устойчиво неэффективной, то есть эффект запирания («lock in» эффект, «институциональная ловушка») не возникнет. Хотя проблема представляется в том, как определить «ловушечное состояние» института (нормы), что считать под его/ее неэффективностью и устойчивостью

На рисунке 5 слева показаны варианты роста дисфункции института, агента или системы, в зависимости от дисфункции по одной, двум, трем, четырем и пяти базисным функциям (общее число функций равно пяти $-F_{\mathrm{O}}=5$ ). Применительно к системе управления под этими функциями можно понимать организацию, планирование, контроль, мотивацию и координацию. Конечно, дисфункция даже од-

\footnotetext{
1 Термины институт, правило, норма употребляются здесь равнозначно, просто для удобства стилистических оборотов подачи текста.
} 

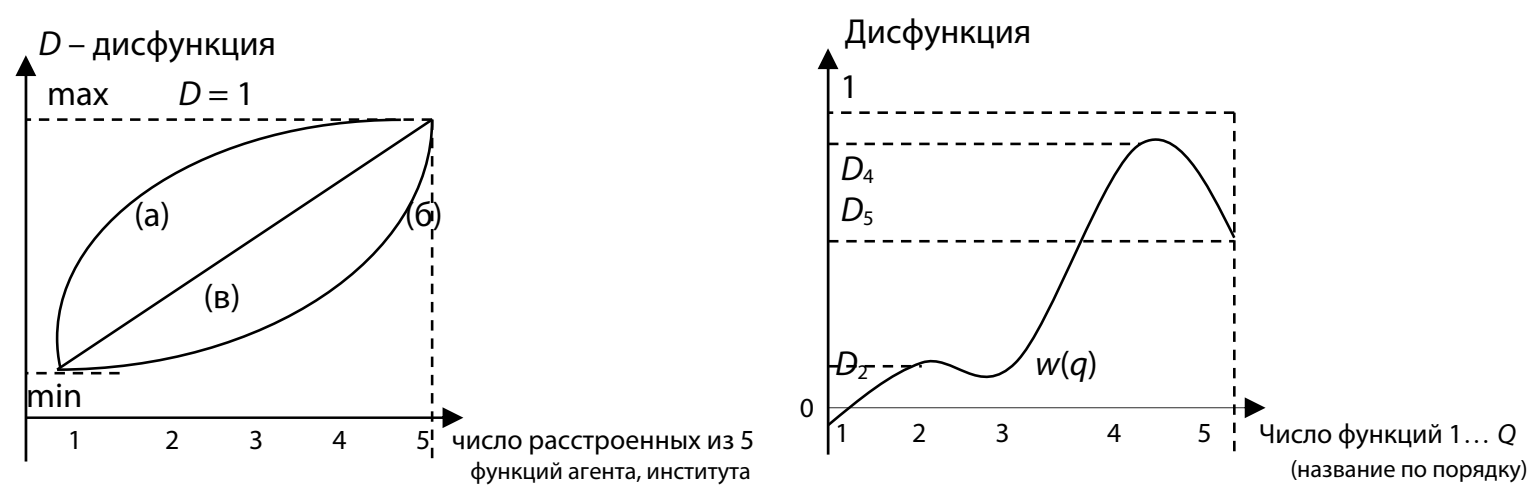

Рис. 5. Функции агента, объекта, института и общая дисфункция

ной функции может оказаться настолько сильной, что приведет к полному «параличу» управления. График на рисунке 5 показывает рост дисфункции по мере увеличения расстроенных функций, так что когда все пять функций расстроены, то дисфункция этого института или системы будет максимальной (равна единице). Выпуклость-вогнутость кривой говорит о чувствительности различных функций, вносящих при их расстройстве вклад в общую величину дисфункции. Для какой-то системы кривая может быть представлена в виде линий (а) и (б), для другой - в виде линии (в), отражающей планомерное нарастание дисфункции. Конечно, рисунок 5 отражает не все возможные варианты, ибо рост общей дисфункции системы может быть стабилизирован ниже максимально возможного уровня по причине снижения глубины дисфункции предыдущих функций к тому моменту, когда возникла дисфункция пятой функции. К тому же функции расстраиваются не последовательно, а выборочно, и вносят разный вклад в общую дисфункцию. Если имеется функциональная связь, то расстройство одной функции может приводить к расстройству другой функции, и тогда уровень дисфункции института или системы быстро нарастает в силу наличия такой связи между функциями.

Институт или система, исполняющие большое число функций, могут характеризоваться различной величиной вклада в обеспечение дисфункции. Например, 10 \% от общего числа одних функций дают минимальную степень дисфункции, но другие 10 \% функций обеспечивают 80 \% текущей дисфункции. Чтобы учесть такое распределение вклада, можно воспользоваться распределением М.О. Лоренца, которое тот применил к оценке уровня неравенства в распределении дохода ${ }^{1}$. Вместе с тем

\footnotetext{
${ }^{1}$ Если для какого-то института, агента, системы возможно построить распределение Лоренца применительно к шкале доли функций и степени дисфункции, тогда, по аналогии с коэффициентом Джини, не представляет большой слож-
}

каждая функция расстроена по-своему, как бы ни были связаны функции и от чего бы это ни зависело. Поэтому профиль дисфункции покажет соотношение расстройства по крайней мере базисных функций института, агента, системы (см. рисунок 5, справа). Наиболее сильно не выполняются функции 4 и 5 на рисунке 5 , справа. Самая низкая дисфункция функции 1-й, а также близка дисфункция 2-3-й функций. Конечно, ряд функций может быть в принципе не расстроен, исполняться, так как без их исполнения невозможно представить существо работы системы. Тогда профиль охватит оси абсцисс с нулевой дисфункцией. Площадь фигуры, очерчиваемой линией $w(q)^{2}$, осью абсцисс и вертикальными линиями, задаваемыми слева ординатой, справа - заключительной из общего числа $Q$ (натуральное число) функций института (в примере пять функций), обозначает общую величину дисфункции. Можем записать:

$$
D=\int_{1}^{Q} w(q) d q
$$

либо

$D=\frac{1}{Q} \sum_{i=1}^{Q} d_{i} \leq 1$, где $d_{i}-$ дисфункции $i$-й функции.

Безусловно, снижение дисфункции по отдельным функциям закономерно должно снижать общую величину дисфункции агента, института, системы, тем самым повышая исполнение релевантных функций и повышая качество исполнения функций, а также институциональную эффективность. Хотя рост числа исполняемых функций может приводить к непропорциональному росту трансакционных издержек, так что общая институциональная эффективность может не возрасти, а даже

ности измерить дисфункцию аналогичным образом.

${ }^{2}$ Где $q$ - номер функции института из общего числа функций $Q$. 
снижаться. К тому же отдельные функции могут вносить настолько значимый вклад в дисфункцию, что снижение расстройства по иным функциям кардинально не изменит степень дисфункции.

Кроме того, динамика дисфункции по каждой функции института или агента в общем случае может быть связана с динамикой дисфункции по иным функциям. Такие связи требуют больших исследовательских усилий при изучении конкретных объектов и ситуаций (институтов и подсистем). Для простоты анализа можно рассматривать отсутствие тесной связи между дисфункциями по различным функциям института или агента.

Таким образом, как было показано, институт или система характеризуются некоторой степенью дисфункции, которая динамически изменяется в силу разных причин. Благосостояние экономики не может не зависеть от того, насколько расстроены исполняемые институтами и агентами функции - связь эта такая же нелинейная, как и эффективности институтов и их дисфункции.

Рассмотрим экономику коузинского типа (Coase, 1988), состоящую из двух агентов, например фермера, выращивающего пшеницу, и скотовода, производящего мясо. Дадим основные функции этих агентов (см. табл. 1), необходимые им для того, чтобы создать продукцию и обеспечить себе тем самым некоторый уровень благосостояния. Причем благосостояние такой экономики складывается из продукта, создаваемого фермером $\left(P_{f}\right)$ и скотоводом $\left(P_{s}\right)$, то есть $P=P_{f}+P_{s}$.

Как следует из таблицы 1 , если фермер и скотовод являются соседями, то может возникнуть конфликт в производстве продуктов, то есть в создании благосостояния экономики. Он развертывается по функции (3), когда скотовод выгоняет стадо на выпас, тем самым вытаптывая посевы пшеницы, которые охраняются фермером. Причем данная функция поначалу может вообще отсутствовать, так как агент, в данном случае фермер, может не представлять в исходной точке производства опасность, исходящую от скотовода. По этой причине функция охраны возникает как добавочная функция, когда налицо ущерб посевам от выпаса скота. Подобная ситуация возникает как раз потому, что не определено право на прогон скота, а сам конфликт порождает трансакционные издержки вследствие неопределения права собственности на прогон. При этом, нужно заметить, что право собственности на пастбище, поля под пшеницу - определено, как и на другие виды деятельности, и закрепление функций за агентами. Ущерб фермера оборачивается выгодой скотовода, но величина ущерба в общем случае не равна величине выгоды. Причина в том, что механические повреждения посевов зависят от величины стада, характера прогона скота и многих сопровождающих этот процесс условий. К тому же урожай пшеницы зависит от погодных условий, как и возможность восстановиться растениям после того, как по ним прошел скот. Поэтому общее благосостояние экономики, определяемое суммой создаваемых продуктов, может увеличиться, уменьшиться или не измениться. Это будет зависеть от того, насколько сократится урожай пшеницы и увеличится объем мяса в силу хорошего питания стада при прогоне на пастбище. Чтобы предотвратить ущерб, фермер может ввести функцию 3 - охраны посевов, если издержки на охрану меньше ущерба, который возникает при прогоне стада скотоводом. Однако реализация функции 3 фермером связана с тем, чтобы запретить прогон стада скотоводом, то есть, распространяется на случай, когда право собственности на прогон не определено и скотовод не обладает этим правом. Если функция 3 введена, либо присутствовала изначально, тем не менее, она может исполняться не на должном уровне, быть подвергнутой дисфункции. В этом случае какой-то ущерб принимает на себя фермер именно в силу дисфункции. Если право собственности не определено, эту ситуацию также можно рассматривать как вариант дисфункции ин-

Таблица 1

Основные функции при производстве пшеницы и мяса

\begin{tabular}{|c|l|l|}
\hline $\begin{array}{c}\text { Номер } \\
\text { функции }\end{array}$ & $\begin{array}{c}\text { Содержание функции производства пше- } \\
\text { ницы - фермера }\end{array}$ & $\begin{array}{c}\text { Содержание функции производства мяса - } \\
\text { скотовода }\end{array}$ \\
\hline 1 & Культивация земли (подготовка к посеву) & Подготовка пастбища, приобретение поголовья \\
\hline 2 & Посев пшеницы & Размещение стада, уход \\
\hline 3 & Охрана посевов & Выпас скота на пастбище \\
\hline 4 & Уход и поддержание роста & Забой, получение мяса, шерсти \\
\hline 5 & Сбор урожая, хранение и переработка & Хранение, продажа продукции \\
\hline
\end{tabular}




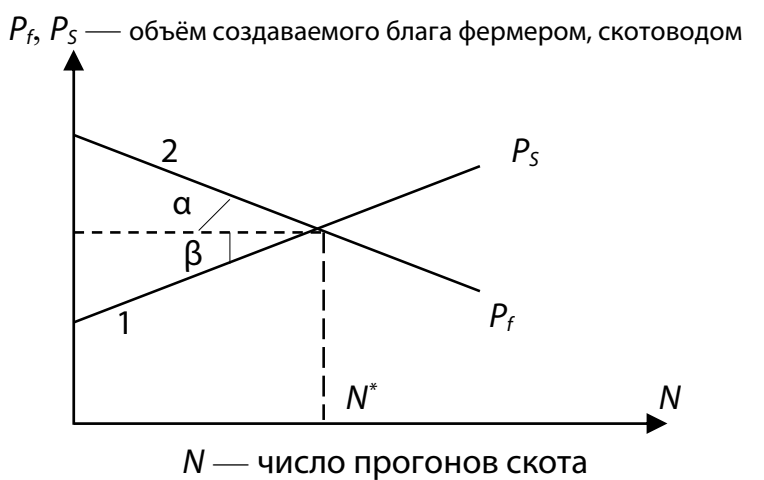

Рис. 6. Изменение благосостояния экономики фермера - скотовода, $P=P_{f}+P_{s}$

ститута «права собственности», поскольку при неопределении права неясно распределение функций, ответственности и т.д. Если право собственности определено, то размещение ресурсов не изменяется, как и структура производства, однако до того момента, когда произойдет изменение права собственности. Проблема не только в том, что изменение права собственности приведет к росту трансакционных издержек, которые можно считать низкими, пока право собственности четко определено, но и в том, что изменение права собственности означает смену владельца, с вытекающим влиянием на производственный процесс, даже если трансакционные издержки равны нулю. При нулевых трансакционных издержках трансформационные издержки нулю точно не равны, и они могут быть зависимы не только от того, кто реализует право собственности - функции владения и управления, но и от степени дисфункции. Разной степени дисфункции будет отвечать различная эффективность производства при смене права собственности. Это не сможет не оказать влияния на уровень благосостояния и структуру производства. Предпримем некоторые формальные построения для экономики из двух агентов фермера и скотовода, причем для случая, когда скотовод наносит ущерб фермеру, тем самым выступая для него своеобразным генератором внешнего эффекта. Развитие экономики описывает рисунок 6,

Как видим, в зависимости от числа прогонов скота, вытаптывающего посевы фермера, объем создаваемого скотоводом блага увеличивается, а фермера, поскольку посевы вытаптываются, сокращается. Общее благосостояние измеряется суммой $P=P_{f}+P_{s}$, структура производства $s=P_{s} / P_{f}$.

В точке $N^{*}$ для данного числа прогонов скота создаваемое благо двух агентов сравняется (эту точку считаем неизменной характе- ристикой данной экономической системы). Исходное значение таково, что фермер создавал больший объем блага, когда число прогонов скота было равно нулю, но затем ситуация динамически изменяется. Причем экономика явно претерпевает структурное изменение, так как изменяется отношение $P_{s}$ к $P_{f}$ в силу изменяемого объема создаваемого блага каждым агентом. Связь производства двух видов благ задается, согласно рисунку 6, следующим образом: $P_{f}=P_{s}+k\left(N^{*}-N\right)$, откуда общее благосостояние будет $P=2 P_{s}+k\left(N^{*}-N\right)$, где $k=(\operatorname{tg} \alpha+$ $+\operatorname{tg} \beta)$. Исходим из того, что чувствительность создаваемого блага к числу прогонов скота не изменяется для каждого агента и задается углом наклона его линии создания блага (линии 1-2 на рисунке 6). Изменение благосостояния экономики двух агентов составит: $d P / d t=$ $=2 d P_{s} d t-k d N / d t$. Увеличение благосостояния $d P / d t>0$, если оно не изменяется $d P / d t=0$. Откуда темп роста создаваемого скотоводом блага $g_{s}=\left(1 / P_{s}\right) d P_{s} / d t$ должен превышать взвешенное изменение числа прогонов скота, то есть $g_{s}>\left(k / 2 P_{s}\right) d N / d t$. Конечно, в идеале необходимо наложить условие на изменение создаваемого блага фермером, которое в общем случае не должно сокращаться. Если благосостояние экономики не изменяется, то коэффициент структуры $s=-g_{f} / g_{s}$. При росте благосостояния $s>-g_{f} / g_{s}$.

Если создаваемое благо скотоводом и фермером складывается из совершаемых трансакций (трансакционных издержек $-\operatorname{Tr}_{s}, \operatorname{Tr}_{f}$ - соответственно скотовода и фермера ) и полученной прибыли $\left(p_{r s}, p_{r f}\right)$, то есть, $P_{s}=\operatorname{Tr}_{s}+p_{r s}, P_{f}=T r_{f}+p_{r f}$, то трансакционные издержки можно представить как некую долю создаваемого блага каждым агентом: $\operatorname{Tr}_{s}=a P_{s}, \operatorname{Tr}_{f}=b P_{f}, a, b-$ коэффициенты пропорциональности, тогда несложно показать, учтя, что $1-a=r_{s}, 1-b=r_{f}-$ нормы прибыли скотовода и фермера, что:

$$
\begin{aligned}
& P_{s}=\frac{p_{r s}}{1-a} ; P_{f}=\frac{p_{r f}}{1-b}, \\
& P=\frac{2 p_{r s}}{1-a}+k\left(N^{*}-N\right) .
\end{aligned}
$$

Исходя из приведенных соотношений, можно получить ограничение на темп роста прибыли скотовода при условии, чтобы благосостояние экономики увеличивалось $d P / d t>0$ и доля трансакционных издержек в создаваемом благе изменяется. Тогда темп роста прибыли должен превышать разницу взвешенных темпа роста числа прогонов скота и темпа роста доли трансакционных издержек: 


$$
g_{r s}>\frac{k N}{2 P_{s}} g_{N}-\frac{a}{1-a} g_{a} .
$$

где $g_{N}=(1 / N) d N / d t ; g_{a}=(1 / a) d a / d t ; g_{r s}=$ $=\left(1 / p_{r s}\right) d p_{r s} / d t$.

Если скотовод и фермер стремятся к наибольшей прибыли, то несложно определить величину создаваемого блага, приносящего наибольшую прибыль.

Прибыль скотовода и фермера, соответственно: $p_{r s}=P_{s}(1-a) ; p_{r f}=P_{f}(1-b)$. Условие наибольшей прибыли $d p_{r s}=0, d p_{r f}=0$. Откуда получаем величину создаваемого блага, отвечающего наибольшей прибыли $\left(c_{1}, c_{2}-\right.$ константы интегрирования) ${ }^{1}$ :

$$
P_{s}=\frac{\exp \left(c_{1}\right)}{1-a}, P_{f}=\frac{\exp \left(c_{2}\right)}{1-b} .
$$

Величина благосостояния экономики $P=$ $=P_{s}+P_{f}$, соответствующая наибольшей прибыли каждого агента:

$$
P=\frac{(1-b) \exp \left(c_{1}\right)+(1-a) \exp \left(c_{2}\right)}{(1-a)(1-b)} .
$$

Исполняемые скотоводом и фермером функции зависят от величины создаваемого блага. Предположим, что чем выше величина производимого блага каждым агентом, тем большее число функций необходимо исполнить. Такое допущение предполагает запись $F_{s}=n P_{s}, F_{f}=m P_{f}-$ соответственно число исполняемых функций скотоводом и фермером. Тогда дисфункция скотовода и фермера определится отношением неисполняемых функций к общему числу функций. Общее число функций скотовода и фермера $\left(F_{\mathrm{Os}}, F_{\mathrm{Of}}\right)$ складывается из числа исполняемых $\left(F_{s}, F_{f}\right)$ и неисполняемых $\left(F_{n s}, F_{n f}\right)$ функций: $F_{\text {os }}=F_{s}+F_{n s} ; F_{\text {of }}=F_{f}+F_{n f}$ Тогда дисфункция скотовода $\left(D_{s}\right)$ и фермера $\left(D_{f}\right)$ примет вид, согласно определению в начале статьи:

$$
\begin{gathered}
D_{s}=1-\frac{F_{s}}{F_{\mathrm{Os}}} ; D_{f}=1-\frac{F_{f}}{F_{\mathrm{O} f}} ; \\
D_{s}=1-\frac{n P_{s}}{F_{\mathrm{Os}}}=1-\frac{n}{x_{s}} ; \\
D_{f}=1-\frac{m P_{f}}{F_{\mathrm{O} f}}=1-\frac{m}{x_{f}} .
\end{gathered}
$$

Как видим, дисфункция ниже в том случае, если выше число исполняемых функций на единицу создаваемого блага $(n, m)$, и выше,

\footnotetext{
${ }^{1}$ При известных ограничениях на знак производной слева и справа от экстремума функции прибыли.
}

если больше общее число функций на единицу создаваемого блага $\left(x_{s}=F_{\mathrm{os}} / P_{s} ; x_{f}=F_{\mathrm{of}} / P_{f}\right)$.

При неизменном общем числе функций, чтобы дисфункция сокращалась, необходимо и достаточно, чтобы темп роста создаваемого блага скотоводом и фермером превышал величину темпа роста числа исполняемых функций на единицу продукта с противоположным знаком, то есть, $g_{s}>-g_{n}, g_{f}>-g_{m}$.

Дисфункцию каждого агента можно определить сокращением числа исполняемых функций, тогда основное условие дисфункции примет вид: $d F_{s} / d t<0, d F_{f} / d t<0$ по скотоводу и фермеру соответственно. Полагая, что число исполняемых функций каждым агентом пропорционально создаваемому ими благу с постоянным коэффициентом пропорциональности $-n, m$, запишем: $d F_{s} / d t=n d P_{s} / d t, d F_{f} / d t=m d P_{f} / d t$. Также имеем: $d P_{f} / d t=d P_{s} / d t-k d N / d t ; d P / d t=$ $=d P_{s} / d t+d P_{f} / d t$. Откуда получаем:

$$
\begin{aligned}
& \frac{d P_{f}}{d t}=\frac{1}{2}\left[\frac{d P}{d t}-k \frac{d N}{d t}\right], \\
& \frac{d P_{s}}{d t}=\frac{1}{2}\left[\frac{d P}{d t}+k \frac{d N}{d t}\right] .
\end{aligned}
$$

Для того, что экономика развивалась, необходимо, чтобы $d P / d t>0$ - благосостояние должно возрастать, при этом дисфункция как отдельных агентов, так и системы в целом должна снижаться. Для роста благосостояния необходимо, чтобы изменение создаваемого блага скотоводом и фермером превосходило взвешенное изменение числа прогонов скота:

$$
\begin{gathered}
\frac{d P_{s}}{d t}>\frac{k}{2} \frac{d N}{d t}, \\
\frac{d P_{f}}{d t}>-\frac{k}{2} \frac{d N}{d t} .
\end{gathered}
$$

Чтобы дисфункция каждого агента снижалась, необходимо превосходство изменения совокупного благосостояния над изменением числа прогонов скота (условие 1 - для скотовода со знаком минус, 2 - условие для фермера):
1) $\frac{d P}{d t}>-k \frac{d N}{d t}$,
2) $\frac{d P}{d t}>k \frac{d N}{d t}$.

При сокращении прогонов скота и снижении ущерба условие на снижение дисфункции становится менее критичным для каждого агента.

Для экономики, состоящей из двух рассматриваемых агентов, имеет смысл, чтобы общая дисфункция не возрастала - по всему набору 
Степень функционального расстройства работы скотовода и фермера

Таблица 2

\begin{tabular}{|c|c|c|}
\hline Номер функции по таблице 1 & $\begin{array}{c}\text { Степень расстройства функ- } \\
\text { ции у скотовода, \% }\end{array}$ & $\begin{array}{c}\text { Степень расстройства } \\
\text { функции у фермера, \% }\end{array}$ \\
\hline 1 & 10 & 20 \\
\hline 2 & 30 & $40(70)$ \\
\hline 3 & $100(50)$ & 0 \\
\hline 4 & 0 & $26(32)$ \\
\hline 5 & 0 & $3,7(3,4)$ \\
\hline Величина дисфункции на данный момент,
\end{tabular}

* Оно определяется исходя из выражения $F=F_{\mathrm{O}}(1-D)$, где $F_{\mathrm{O}}=5-$ общее число функций, $D-$ дисфункция. Поскольку отдельные функции исполняются не полностью, дисфункция задана как процентное измерение степени расстройства функции, то и число исполняемых функций получается с долями, не натуральное число.

функций, реализуемых в системе. Это означает, что $d F_{s} / d t+d F_{f} / d t>0$. Откуда изменение благосостояния должно быть таким: $d P / d t>$ $>k[(m-n) /(m+n)] d N / d t$, превосходить взвешенное изменение числа прогонов скота по посевам фермера.

Обратные знаки приведенных неравенств будут говорить об условии, когда дисфункция по агентам и в системе будет увеличиваться, то есть число исполняемых функций, при том же общем числе функций, сокращаться.

В рассмотренном примере на благосостояние оказывают влияние:

- число прогонов скота;

- соотношение числа исполняемых функций к общему числу функций агентов (дисфункция);

- институциональная эффективность, зависящая от величины трансакционных издержек как некой доли от создаваемого блага;

- величина создаваемого блага;

- степень чувствительности создаваемого каждым агентом блага к числу прогонов скота скотоводом;

- степень нанесенного ущерба фермеру, которая сразу отражена в виде линии изменения величины создаваемого блага (линия 2 на рисунке 6).

Допустим, функциональные расстройства скотовода и фермера заданы величинами таблицы 2 (исходный профиль дисфункции - см. рис. 7). Из таблицы 2 следует, что у фермера степень общей дисфункции несколько ниже, чем у скотовода, для данного момента времени. Причем, учитывая, что функция выпаса стада у скотовода расстроена на 100 \%, можно предполагать, что реализуется право на запрет прогона скота. Если это право отменить и предоставить возможность скотоводу прогонять скот, нанося ущерб фермеру, то дисфункция третьей функции у скотовода понизится, скажем, до 50 \%, а у фермера - возрастет, например, до 70 \% (показано в скобках в таблице 2). Тогда общая величина дисфункции изменится (в скобках нижняя строка таблицы 2). Для скотовода дисфункция понизится, для фермера - возрастет.

С ростом дисфункции фермера число исполняемых функций понизится. При тех же трансакционных издержках институциональная эффективность снизится, если они возрастут, то снижение эффективности будет еще более значительным. Поскольку число исполняемых функций скотовода при снижении дисфункции возрастет, то при тех же трансакционных издержках институциональная эффективность увеличится. Она может возрасти даже при некотором увеличении этих издержек.

Возможность прогонять скот будет способствовать увеличению создаваемого блага скотоводом, и понижать благо, создаваемое фермером. Это повлияет на величину благосостояния и на структуру производства.

Располагая функцией для создаваемого скотоводом и фермером блага от числа прогонов

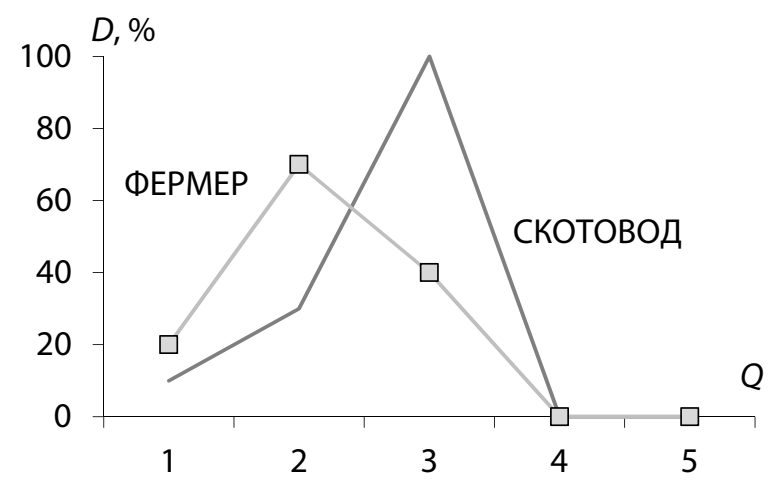

Рис. 7. Исходный профиль дисфункции скотовода и фермера 
скота (по рис. 6) $P_{s}=f_{s}(N), P_{f}=f_{f}(\mathrm{~N})$, учитывая связь трансакционных издержек с создаваемым благом $T r_{s}=a P_{s}, T r_{f}=b P_{f}$, несложно дать оценку институциональной эффективности, представленной отношением числа исполняемых функций к величине трансакционных издержек: $E_{I s}=F_{s} / \operatorname{Tr}_{s}, E_{I f}=F_{f} / \operatorname{Tr}_{f}$. Тогда можем записать: $E_{I s}=F_{s} /\left[a f_{s}(N)\right], E_{I f}=F_{f} /\left[b f_{f}(N)\right]$. В случае, когда число исполняемых функций пропорционально создаваемому благу $F_{s}=n P_{s}, F_{f}=m P_{f}$, институциональная эффективность составит: $E_{I s}=n / a, E_{I f}=m / b$.

Если доля трансакционных издержек скотовода и фермера одинакова от величины продукта, но продукт фермера больше пока $N<N^{*}$, то институциональная эффективность фермера ниже по величине, так как число исполняемых функций меньше, а трансакционные издержки выше, чем у скотовода (для рассматриваемого примера таблицы 2).

Теорема Коуза (Coase, 1988), утверждающая, что если изначально право собственности определено (земля для посева и пастбище принадлежат соответственно фермеру и скотоводу, а вот право на прогон скота - может принадлежать скотоводу, либо на запрет такого прогона - фермеру, либо ситуация может оказаться неопределенной $\left.{ }^{1}\right)$ и трансакционные издержки отсутствуют, то изменение права собственности не повлияет на структуру производства и даже на его эффективность (Stigler,1966. Р. 113; Lai, 2011. P. 45). Вместе с тем, смена права собственности, изменение правовой позиции, по Коузу, приводит к изменению функционального набора, так как актив, на который распространяется право, характеризуется своим функциональным набором. Разница в исходной институциональной эффективности агентов, а также степени дисфункции, имеют принципиальное значение при анализе вопроса распределения права собственности. Исходная дисфункция агентов также различается, следо-

\footnotetext{
${ }^{1}$ В последнем случае фермер нанимает охрану для того, чтобы защитить свои посевы, если затраты на содержание охраны не превышают ущерб, который наносится посевам и урожаю от прогона скота, когда посевы не охраняются. Проблема в том, что на урожай действует масса иных факторов, так что определить, как повлияла потрава посева скотом, совсем не просто. Именно издержки измерения и доказательства факта потравы посевов составляют правовые издержки - составляющие трансакционные затраты фермера. Однако, если эти издержки равны нулю, то фермеру проще не нанимать охрану (в этом случае издержки явно не ноль), а выставить ущерб скотоводу, если издержки получения компенсации от скотовода также будут равны нулю (это возможно в случае согласия скотовода с предъявляемыми обвинениями).
}

вательно, восприятие ими и реализация права собственности будут различаться, что даже при нулевых трансакционных издержках, которые при изменении правовой традиции всё-таки нулю не равны (хотя бы по причине различной дисфункции агентов и влияющих на них институтов), тем не менее, даст иное распределение трансформационных издержек ${ }^{2}$. Причем имеется принципиальная разница в правовом смысле между правом на прогон скота, либо отсутствием этого права, либо правом на запрет прогона скота. И эти правомочия реализуются в экономике по-разному, причем каждый агент может по-своему воспользоваться имеющейся институциональной организацией. В связи с этим даже при нулевых трансакционных издержках и при исходном определении права собственности изменение правовой позиции агента повлияет на результат производства и структуру. Такой исход будет характерен для экономики «не классной доски», а для экономики, где агенты соревнуются по использованию институтов, где они, как и институты, в различной степени подвержены дисфункции, да и обладают отличающимся функциональным потенциалом. По этой причине их собственные трансакционные издержки отличаются, что не может не оказать воздействие на издержки их контрактации. Конечно, речь идет об агентах, настроенных извлекать дополнительную выгоду от трансакций и намеренного влияния с этой же целью по поводу изменения действующих правил. Ситуация осложняется тем, что дисфункция институтов и агентов возникает как в области неэффективности, так и эффективности, хотя наименьшая эффективность все-таки соответствует большему значению дисфункции. Не может быть значительная часть функций института расстроена, а он при этом показывать наибольшую эффективность функционирования. С ростом дисфункции возможности институциональных изменений ограничиваются. Так, если фермер имеет высокую дисфункцию по функциям $1-2$ (табл. 1 ), то обеспечение функции 3 - охраны посевов может также пострадать вследствие, например, снижения мотива реализации этой функции. Если охрана посевов осуществляется ферме-

\footnotetext{
2 Получается парадокс, что «теорема» верна только для трансакционной деятельности, да ещё такого её случая, когда трансакционные издержки равны нулю. А в этом случае такая деятельность должна отсутствовать, да и распределение права при нулевых издержках в обществе трудно себе представить. При ненулевых трансакционных издержках распределение права должно повлиять на ценность производства и его структуру.
} 
ром слабо, то этой ситуацией воспользуется скотовод для увеличения производства его блага, конечно, если у скотовода имеется мотив на достижение такой цели. При действии права на запрет прогона скота у скотовода функция 3 является неисполняемой и дисфункция равна 0,2 при исполнении оставшихся четырех функций (табл. 1). У фермера при исполняемых всех пяти функциях дисфункция может отсутствовать. Запрет на прогон скота понижает возможность для скотовода увеличивать создаваемое им благо. Если право на запрет прогона скота отсутствует, то фермер может усилить охрану своих посевов. Тогда от того, как именно выполняется эта функция и каковы последствия для скотовода от ее исполнения (судебные решения), при неравенстве нулю трансакционных издержек, будут зависеть результат производства блага у скотовода и фермера и изменение общей величины благосостояния данной экономической системы.

Изменение права собственности на прогон скота, принадлежит ли оно скотоводу, либо право на запрет принадлежит фермеру, конечно, повлияет на результат производства и изменение благосостояния, как и показано выше. Дисфункция самого этого права как института будет различной относительно того, кому это право принадлежит, и допущение о нулевых трансакционных издержках с практической точки зрения здесь выступает чрезмерно сильным ограничением, делающим экономическую конструкцию далекой от жизни. Если пренебречь дисфункцией института, считая его совершенным, тогда и трансакционные издержки можно положить равными нулю, но и в этом случае надо отключить иные факторы и функции, чтобы в чистом виде рассматривать только один вид правовой позиции. Однако в реалиях право собственности не распространяется дискретно и рассматривается агентом отдельно, оно образует общий континуум правомочий, затрагивающих владение средствами производства и иными активами, используемыми в процессе создания благ, что сказывается на изменении общего благосостояния системы. Оно в свою очередь зависит и от режима контрактации агентов, составляющих экономику, взаимных реакций и связности благ. Эти и многие иные условия коренным образом приводят к закономерному расширению теоремы Коуза - распространяя ее только на часть «трансакционных активов», отрывая одну правовую позицию, к которой применяются допущения теоремы, от всех остальных.
Случай же ненулевых трансакционных издержек закономерно предполагает иную формулировку теоремы (Сухарев, 2017. С. 56-57), а именно сводимую к тому, что распределение права собственности будет влиять на ценность и структуру производства.

\section{Заключение}

Подведем итог проведенному анализу. Вопервых, понимаемая как расстройство (либо потеря) функций дисфункция правил, агентов, систем может наблюдаться при различной величине институциональной эффективности, причем наибольшей эффективности не всегда соответствует минимальная дисфункция.

Во-вторых, функциональный анализ сделок (контрактации), функционирования экономики позволяет расширить представления о благосостоянии и его изменении. В частности, исходная институциональная эффективность и степень дисфункции агентов контрактации напрямую влияют на уровень и динамику благосостояния. В связи с этим распределение права собственности как институциональная коррекция взаимодействия изначально в различной степени дисфункциональных агентов и правил, затрагивающих и право собственности, становятся сильным ограничителем применения теоремы Коуза, которая на случай ненулевых трансакционных издержек не распространяется по факту своей формулировки. В задаче взаимодействия двух агентов (скотовода и фермера) получено условие роста благосостояния отдельного агента и экономики двух агентов, зависящее от числа прогонов скота, что в свою очередь определяется правом на прогон или запрет прогона, то есть институциональными условиями, определяемыми распределением права собственности. Причем нужно рассматривать не только право на прогон, но и право на создание/производства блага, изменение которого без механизма принуждения и соответствующих трансакционных издержек не представляется возможным.

Тем самым аспект связи дисфункции правил и институциональной эффективности представляет центральное направление исследований не только в рамках структурно-функционального анализа институциональной теории, но представляет важность с точки зрения управления, подготовки решений, составляющих существо институциональных коррекций, например, изменения прав собственности либо регулирования экономических отношений относительно таких прав. 


\section{Список источников}

Веблен Т. Теория делового предприятия. - М. : Дело, 2007. - 288 с.

Веблен Т. Теория праздного класса. - М.: Книжный дом «Либроком», 2010.

Клейнер Г. Б. Экономика. Моделирование. Математика. Избранные труды. - М. : ЦЭМИ РАН, 2016.

Мертон Р. К. Социальная теория и социальная структура. - М. : АСТ, Хранитель, 2006.

Полтерович В. М. Трансплантация экономических институтов // Экономическая наука современной России. 2001. - № 3. - С. 24-50.

Сухарев О. С. Эволюционная экономическая теория институтов и технологи. Проблемы моделирования. - М. : Ленанд, 2017.

Сухарев О.С. Экономический рост, благосостояние и институциональные изменения // Журнал институциональных исследований. - 2011. - Т. 3. - № 3. - С. 19-39.

Сухарев О. С. Теория экономической дисфункции. - М.: Машиностроение-1, 2001.

Сухарев О. С. Российская экономика: опыт системной диагностики и лечения // Инвестиции в России. -1999. - № 9. - C. 28-37.

Bartolini D., Santolini R. Political institutions behind good governance // Economic Systems. — 2017. — Vol. 41. — Issue 1. - P. 68-85.

Beverelli C., Fiorini M., Hoekman B. Services trade policy and manufacturing productivity: The role of institutions // Journal of International Economics. — 2017. - Vol. 104. - P. 166-182.

Chan S.-G., Koh E., Zainir F., Yong C.-C. Market structure, institutional framework and bank efficiency in ASEAN 5 // Journal of Economics and Business. - 2015. - Vol. 82. - P. 84-112.

Coase R. Essays on Economics and Economists. - University of Chicago Press, 1994. - 232 p.

Coase R. The Firm, the Market, and the Law. - University of Chicago Press, 1988. - 217 p.

Davanzati G. F. Structural change driven by institutions: Thorstein Veblen revised // Structural Change and Economic Dynamics. - 2018. - Vol. 45. - P. 105-110.

Eggertsson T. Knowledge and the Theory of Institutional Change // Journal of Institutional Economics. - 2009. Vol. 5(2). - P. 137-150.

Hartwell C. A. The institutional basis of efficiency in resource-rich countries // Economic Systems. - 2016. - Vol. 40.

- Issue 4. - P. 519-538.

Hodgson G. Economic Evolution. - University of Chicago Press, 2010. — 294 p.

Khalil E. L. Lock-in institutions and efficiency // Journal of Economic Behavior \& Organization. - 2013. - Vol. 88. P. 27-36.

Lai L. W. The ideas of Ronald H. Coase: Market failure and planning by contract for sustainable development. — London : Routledge. $-2011 .-312 \mathrm{p}$.

Leeuw T., Gössling T. Theorizing change revisited: An amended process model of institutional innovations and changes in institutional fields // Journal of Cleaner Production. - 2016. - Vol. 135. - P. 435-448.

$\mathrm{Ma}$ T.-C. Competition authority independence, antitrust effectiveness, and institutions // International Review of Law and Economics. - 2010. - Vol. 30. - Issue 3. - P. 226-235.

Mahoney J., Thelen K. A. Gradual Theory of Institutional Change. Explaining Institutional Change: Ambiguity, Agency, and Power. - Cambridge: Cambridge University Press, 2010.

Mohamad N. Telecommunications reform and efficiency performance: Do good institutions matter? // Telecommunications Policy. - 2014. - Vol. 38. - Issue 1. - P. 49-65.

North D. Understanding the Process of Economic Change. - Princeton University Press, 2005. - $187 \mathrm{p}$.

North D. Institutional Change and American Economic Growth. - Cambridge University Press, 2008. - 292 p.

North D. Institutions, Institutional Change, and Economic Performance. - Cambridge University Press, 1990. - 152 p.

Stigler G. The Theory of Price. - New York: Macmillan Library Reference, 1966. - 355 p.

Stiglitz J. Creating a Learning Society: A New Approach to Growth, Development, and Social Progress. - Columbia University Press, 2014. - 680 p.

Williamson O. The Theory of the Firm as Governance Structure: From Choice to Contract // Journal of Economic Perspectives. - 2002. - No. 16 (3). - P. 171-195.

Yang H., Jung W.-S. Structural efficiency to manipulate public research institution networks // Technological Forecasting and Social Change. - 2016. - Vol. 110. - P. 21-32.

\section{Информация об авторе}

Сухарев Олег Сергеевич - доктор экономических наук, профессор, главный научный сотрудник, Институт экономики Российской академии наук (Москва, Российская Федерация; e-mail: o_sukharev@list.ru). 
For citation: Sukharev, O. S. (2020). Dysfunction of the Rules and Institutional Effectiveness. Zhurnal Economicheskoj Teorii [Russian Journal of Economic Theory], 17 (2), 433-450

Sukharev O. S. Institute of Economics of the Russian Academy of Sciences (Moscow, Russian Federation; e-mail: o_sukharev@list.ru)

\section{Dysfunction of the Rules and Institutional Effectiveness}

The purpose of the study is to determine the possible relationship of the institutional effectiveness, which is understood as the number of executable functions per unit of transaction costs and dysfunction understood as the disorder, non-performance a certain number of functions arising for various reasons in respect to institutions, agents, systems, as well as individual functions. I use the structural-functional research method, which allows to give the measuring interpretation at the theoretical level of analysis, based on the definition of concepts, as well as at the formal level to relate the expected change of the studied values. I propose the theoretical model of connection between dysfunction and institutional effectiveness, which shows that the disorder of the introduced rules' functions may reduce the quality of functioning. But some efficiency will be ensured at the same time. Some of the rules are executed for this level of costs. On that basis, the frequency and content of changes in institutions affect the quality of development, changes in the level of welfare of both individual agents, and the economy as a whole. This effect is shown in the example used by $R$. Coase in substantiating the 'theorem' formulated by G. Stigler, namely the interaction between a cattleman and a farmer, when running the herd on crops causes damage to the farmer, acting as the negative externality. There is considered the economy of two agents, whose welfare depends on how many times they drive the cattle out, the distribution of ownership to the girder, the initial and changeable values of institutional effectiveness, and the dysfunction of agents. I have obtained the result that the largest value of institutional effectiveness does not correspond to the smallest dysfunction value in all cases, with the considered method of determining these parameters. I propose the method for assessing the dysfunction profile of an institution or agent, expanding the possibilities of applying functional analysis within the framework of institutional economic theory. I show the possible relationship between the magnitude of the benefits created by interacting agents and institutional effectiveness. It is shown that even the equal-zero situation of transaction costs does not take into account the 'functional competence' of agents that can affect the effectiveness of the property rights used. Therefore, in this case, the distribution of rights will affect the value and structure of production. In the case of non-zero transaction costs, this effect becomes decisive in assessing the dynamics of welfare and the effectiveness of the contractor.

Keywords: rules, function, dysfunction, adaptation, institutional effectiveness, correction of institutions, welfare agents, structural and functional analysis.

\section{References}

Veblen, T. (2007). Teoriya delovogo predpriyatiya [The theory of business enterprise]. Moscow, Russia: Delo, 288. (In Russ.)

Veblen, T. (2010). Teoriya prazdnogo klassa [The theory of the leisure class]. Moscow, Russia: Knizhnyy dom «Librokom». (In Russ.)

Kleiner, G. B. (2016). Ekonomika. Modelirovanie. Matematika. Izbrannye Trudy [Economic and mathematical methods and economic theory]. Moscow, Russia: TsEMI RAN. (In Russ.)

Merton, R. K. (2006). Sotsial'naya teoriya i sotsial'naya struktura [Social theory and social structure]. Moscow, Russia: AST, Khranitel'. (In Russ.)

Polterovich, V. M. (2001). Transplantatsiya ekonomicheskikh institutov [Transplantation of Economic Institutions]. Ekonomicheskaya nauka sovremennoy Rossii [Economics of Contemporary Russia], 3, 24-50. (In Russ.)

Sukharev, O. S. (2017). Evolyutsionnaya ekonomicheskaya teoriya institutov i tekhnologi. Problemy modelirovaniya [Evolutionary economic theory of institutions and technologies. Problems of modeling]. Moscow, Russia: Lenand. (In Russ.)

Sukharev, O. S. (2011). Ekonomicheskiy rost, blagosostoyanie i institutsional'nye izmeneniya [Economic growth, welfare and institutional change]. Zhurnal institutsional'nykh issledovaniy [Journal of Institutional Studies], 3(3), 19-39. (In Russ.)

Sukharev, O. S. (2001). Teoriya ekonomicheskoy disfunktsii [Theory of economic dysfunction]. Moscow, Russia: Mashinostroenie-1. (In Russ.)

Sukharev, O. S. (1999). Rossiyskaya ekonomika: opyt sistemnoy diagnostiki i lecheniya [Russian economy: experience of system diagnostics and treatment]. Investitsii v Rossii [Investment in Russia], 9, 28-37. (In Russ.)

Bartolini, D., \& Santolini, R. (2017). Political institutions behind good governance. Economic Systems, 41(1), $68-85$.

Beverelli, C., Fiorini, M., \& Hoekman, B. (2017). Services trade policy and manufacturing productivity: The role of institutions. Journal of International Economics, 104, 166-182.

Chan, S.-G., Koh, E., Zainir, F., \& Yong, C.-C. (2015). Market structure, institutional framework and bank efficiency in ASEAN 5. Journal of Economics and Business, 82, 84-112.

Coase, R. (1994). Essays on Economics and Economists. University of Chicago Press, 232.

Coase, R. (1988). The Firm, the Market, and the Law. University of Chicago Press. 217.

Davanzati, G. F. (2018). Structural change driven by institutions: Thorstein Veblen revised. Structural Change and Economic Dynamics, 45, 105-110.

Eggertsson, T. (2009). Knowledge and the Theory of Institutional Change. Journal of Institutional Economics, 5(2), 137150. 
Hartwell, C. A. (2016). The institutional basis of efficiency in resource-rich countries. Economic Systems, 40(4), 519-538. Hodgson, G. (2010). Economic Evolution. University of Chicago Press, 294.

Khalil, E. L. (2013). Lock-in institutions and efficiency. Journal of Economic Behavior \& Organization, 88, 27-36.

Lai, L. W. (2011). The ideas of Ronald H. Coase: Market failure and planning by contract for sustainable development. London: Routledge, 312.

Leeuw, T., \& Gössling, T. (2016). Theorizing change revisited: An amended process model of institutional innovations and changes in institutional fields. Journal of Cleaner Production, 135, 435-448.

Ma, T.-C. (2010). Competition authority independence, antitrust effectiveness, and institutions. International Review of Law and Economics, 30(3), 226-235.

Mahoney, J., \& Thelen, K. A. (2010). Gradual Theory of Institutional Change. Explaining Institutional Change: Ambiguity, Agency, and Power. Cambridge: Cambridge University Press.

Mohamad, N. (2014). Telecommunications reform and efficiency performance: Do good institutions matter? Telecommunications Policy, 38(1), 49-65.

North, D. (2005). Understanding the Process of Economic Change. Princeton University Press, 187.

North, D. (2008). Institutional Change and American Economic Growth. Cambridge University Press, 292.

North, D. (1990). Institutions, Institutional Change, and Economic Performance. Cambridge University Press, 152.

Stigler, G. (1966). The Theory of Price. New York: Macmillan Library Reference, 355.

Stiglitz, J. (2014). Creating a Learning Society: A New Approach to Growth, Development, and Social Progress. Columbia University Press, 680.

Williamson, O. (2002). The Theory of the Firm as Governance Structure: From Choice to Contract. Journal of Economic Perspectives, 16 (3), 171-195.

Yang, H., \& Jung, W.-S. (2016). Structural efficiency to manipulate public research institution networks. Technological Forecasting and Social Change, 110, 21-32.

\section{Author}

Oleg Sergeevich Sukharev - Doctor of Economics, Professor, Institute of Economics of the Russian Academy of Sciences (Moscow, Russian Federation; e-mail: o_sukharev@list.ru). 